\title{
No implant migration and good subjective outcome of a novel customized femoral resurfacing metal implant for focal chondral lesions
}

\author{
Anders Stålman ${ }^{6}$ (D) Olof Sköldenberg ${ }^{5} \cdot$ Nicolas Martinez-Carranza $^{1} \cdot$ David Roberts $^{2} \cdot$ Magnus Högström $^{3}$. \\ Leif Ryd ${ }^{4}$
}

Received: 26 April 2017 / Accepted: 13 November 2017 / Published online: 22 November 2017

(c) The Author(s) 2017. This article is an open access publication

\begin{abstract}
Purpose Managing focal cartilage injuries in the middle-aged patient poses a challenge. Focal prosthetic inlay resurfacing has been proposed to be a bridge between biologics and conventional joint arthroplasty. Patient selection and accurate implant positioning is crucial to avoid increased contact pressure to the opposite cartilage surface. A customized femoral condyle implant for focal cartilage injuries was designed to precisely fit each patient's individual size and location of damage. The primary objective was to assess implant safety profile, surgical usability of the implant and instruments, and implant migration with radiostereometric analysis (RSA).

Methods Ten patients 36-56 years with focal chondral defects, ICRS 3-4 of the femoral cartilage and failed earlier conservative or surgical interventions with VAS pain $>40$. The patients were followed for 2 years with subjective outcome measures (VAS, EQ5D, KOOS) and RSA. The customized implant and guide instruments were manufactured by computer-aided design/computer-aided manufacturing (CAD/CAM) techniques using MRI data.

Results VAS, EQ5D and KOOS showed improvements that reached significance for VAS $(p \leq 0.001)$, Tegner $(p=0.034)$ and the KOOS subscores ADL $(p=0.0048)$, sport and recreation $(p=0.034)$ and quality of life $(p=0.037)$. VAS and KOOS scores improved gradually at 3, 6 and 12 months. The improvements in EQ5D, KOOS pain and KOOS symptoms did not reach statistical significance. No infections, deep venous thrombosis or other complications occured in the postoperative period. No radiographic signs of damage to the opposing tibial cartilage was noted. The surgical usability of implants and instruments were good. RSA did not show any implant migration.

Conclusion This is the first clinical report of a new customized, focal knee resurfacing system. The short-term implant safety and patient-related outcome measures showed good-to-excellent results.
\end{abstract}

Level of evidence Prospective case series, Level 4.

Keywords Focal cartilage injuries · Prosthetic inlay resurfacing · Osteochondral injury

Anders Stålman

anders.stalman@ki.se

1 Department of Orthopaedics, Karolinska University Hospital, Stockholm, Sweden

2 Department of Orthopaedics, Skane University Hospital, Malmö, Sweden

3 Sports Medicine Umeå AB and Orthopedics, Department of Surgical and Perioperative Sciences, Umeå University, Umeå, Sweden
4 LIME, Karolinska Institutet, Solna, Sweden

5 Department of Orthopaedics, Danderyd Hospital, Stockholm, Sweden

6 Stockholm Sports Trauma Research Center, Karolinska Institutet, Capio Artro Clinic, Sophiahemmet, Valhallavägen 91, 11486 Stockholm, Sweden 


\section{Introduction}

Focal cartilage injuries occur frequently in the knee joint and do not heal spontaneously [7, 25, 29]. Patients with focal cartilage defects often have pain and functional impairment that significantly affects quality of life [10]. These lesions might progress to osteoarthritis $[6,18,26]$. Numerous biological treatments, such as microfracture, autologous chondrocyte implantation and mosaicplasty, have been described. Short-term outcome is good especially in the young patient, but the repair tissue might degenerate over time and deteriorating results are seen with increasing patient age. These procedures are therefore not generally recommended for the middle-aged and older patients and treatment options in this group of patients have been insufficient [11, 13-15]. There is a great risk of the need of reintervention after biological treatment [12]. The management of patients who present failed attempts at biological treatment are not well reported in the literature [5]. Unicompartmental or total knee arthroplasties have a high risk of early failure in the younger patient [23].

Managing focal cartilage injuries in the middle-aged patient therefore poses a challenge [19]. Focal prosthetic inlay resurfacing has been proposed to be a bridge between biologics and conventional joint arthroplasty and could be a salvage procedure where biological treatment has failed or is considered less effective [3]. Good short time results in patient-related outcome measures are described but a high rate of revision to knee arthroplasty have been reported [8, 17]. Patient selection and accurate implant positioning is crucial to avoid increased contact pressure to the opposite cartilage surface $[2,20]$.

This new femoral condyle implant for focal chondral injuries together with the guide system is customized and designed to precisely be able to fit each patient's individual size and location of the damage. The double-coated, titanium-hydroxyapatite, Co-Cr implant showed firm and consistent osseochondrointegration in an animal model [21]. The development of a customized prosthesis and guide system made accurate implant positioning possible in order to avoid damage to the opposing cartilage surface [22]. This interventional prospective consecutive cohort study describes the experiences with the first ten patients. The primary objective was to assess implant safety profile, surgical usability of implant and implant migration. The surgical technique is described, short-term outcome evaluated and implant migration is assessed with radiosterometric analysis.

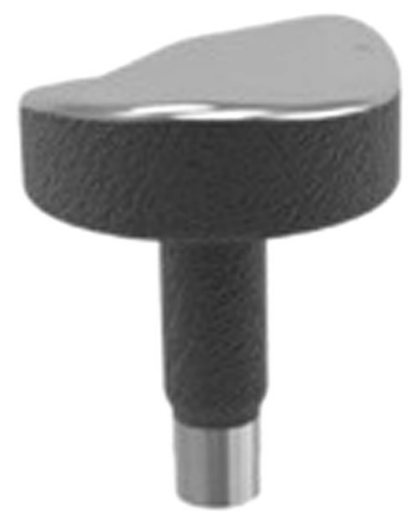

Fig. 1 The Episealer $\mathrm{Cr}-\mathrm{Co}$ implant with a customized circular shaped articulating surface and a hydroxyapatite coated peg for insertion

\section{Materials and methods}

Inclusion criteria were patients 30-65 years with focal chondral defects of the femoral cartilage, International Cartilage Research Society (ICRS) grade 3 or 4 on medial or lateral femoral condyles. The cartilage lesion area was $\leq 3.2 \mathrm{~cm}^{2}$ (diameter $\leq 2 \mathrm{~cm}$ ). The patients had previously failed conservative or surgical interventions such as abrasions, drilling or microfracture and VAS pain $>40$ with activity for more than 6 months. The patients should be capable of completing self-administered questionnaires and willing to comply with the follow-up requirements of the study. Exclusion criteria were BMI $>35 \mathrm{~kg} / \mathrm{m}^{2}$, not addressed instability or other concomitant knee injuries such as meniscus injuries, apart from small flap lesions with intact rim and intact meniscal anterior and posterior horn insertions. Further exclusion criteria were established osteoarthritis, malalignment, metabolic disorders which may impair bone formation, smokers, metal allergies, inflammatory joint diseases, administration of corticosteroids, antineoplastics, immune stimulating or immunosuppressive agents.

\section{Implant}

For this study a customized Cr-Co femoral condyle implant was manufactured by computer-aided design/computer-aided manufacturing (CAD/CAM) technique using MRI data. In addition, from the MRI data specific guide instruments were manufactured as part of the surgical kit to obtain accurate implant positioning in order to avoid damage to the opposing cartilage surface [20]. The $\mathrm{Cr}-\mathrm{Co}$ implant has a 3- to 4-mm-thick circular shape where the articulating surface was contoured to precisely reconstruct each patient's individual cartilage lesion and condylar contour. The implant has a 15 -mm-long and 4-mm-thick peg that was inserted 
in an undersized drill hole to provide primary fixation. For secondary or long-term fixation a double coating of hydroxyapatite-on-top-of titanium was applied on the surfaces facing bone and cartilage (Fig. 1). This coating has been shown to give firm and consistent osseochondrointegration in an animal model [21].

\section{Pre-op planning}

The patient-specific designs of the Episealer implants and surgical drill guides are based upon MRI imaging. The patient undergoes an MRI scan that is uploaded to a MRI processing system $\left(\mu \mathrm{iFidelity}{ }^{\circledR}\right)$ through a web-based online management system. By processing MRI data, a 3-dimensional model of the damaged joint is recreated. The lesion is identified and the implant $\left(\right.$ Episealer $\left.^{\circledR}\right)$ and surgical drill guides (Epiguide ${ }^{\circledR}$ ) are designed to enable the replacement of the damaged area with a fitting implant. The implant is available in four different diameters, 12, 15, 17 and $20 \mathrm{~mm}$, depending on the condyle and lesion size.

\section{Operative technique and description of devices}

The patients were administered cloxacillin $2 \mathrm{~g} \mathrm{15-45} \mathrm{min}$ before surgery and $2 \mathrm{~h}$ postoperatively. Surgery is performed under general or spinal anesthesia. The procedure started with a diagnostic arthroscopy to evaluate the joint and confirm absence of significant concomitant injuries and to validate the size of the injury. The femoral condyle is then exposed through a medial or lateral mini-arthrotomy. After exposure of the femoral condyle, an assessment of the cartilage injury was made ensuring that the implant was sufficiently large to cover the lesion. The drill guide was matched to the unique position on the femoral condyle surface with the lesion centerd in the drill guide. At least two surgical pins should be drilled at the rim of the femoral condyle to firmly fasten the drill guide on the condyle. The rims of the cartilage defect were adjusted with a cartilage cutter and the defect was drilled and milled in order to prepare a hole in the osteochondral tissue for implant insertion. Drill depth was adjusted to counter-sunk the implant $0.5 \mathrm{~mm}$ as indicated by a dummy (Epidummy). The depth was fine-tuned using an adjustment ring in the tool kit allowing incremental adjustment of $0.2 \mathrm{~mm}$ and the final implant position was controlled by a testing device (Epidummy). By inserting the implant, the defect was sealed by the metallic resurfacing implant hence reconstructing the original contour of the femoral condyle. The drillguide and pins were removed and the wound closed in layers. Postoperative pain alleviation by intraarticular ropivacaine was given. Anti-thrombotic medication was not routinely administered. Four different experienced knee surgeons at three different centers performed the surgery and practiced the technique on saw bones prior to the surgery.

\section{Rehabilitation}

Week 1-2: crutches and non-weight-bearing, full passive and active exercises without resistance. Activation of quadriceps muscle in extension. Aims: full passive range of motion.

Week 3-6: crutches and partial weight-bearing. Cycling with light resistance. Straight leg lift. Initiation of corestability training. Leg curls with light resistance. Aims: full active and passive range of motion. Full weight-bearing at week 6.

Week 7-12: full weight bearing, no crutches. Cycling with increasing resistance. Balance training. Squats and lunges with increasing resistance. Aims: normal walking. Good knee control. Managing ADL.

\section{Clinical and radiological evaluation}

Primary endpoints assessments were frequencies of unanticipated side effects such as surgical tools usability and functionality, ongoing osteoarthritis, implant migration, mechanical implant loosening, implant fracture, inflammation, no pain alleviation or allergic reaction. Secondary endpoints assessments were performance compared to baseline by patient reported outcome measurements (PROMS) preoperatively, at 3 and 6 months, 1 and 2 years; Knee injuries and Osteoarthritis Outcome Score (KOOS), EuroQoL (EQ-5D), Tegner Activity Scale (Tegner Score), In addition, Visual Analogue Scale (VAS pain) and knee range of motion (ROM) measurements were recorded.

\section{Radiostereometric analysis (RSA)}

RSA is a high-precision method of assessing three-dimensional (3D) micro-movement from calibrated stereoradiographs and is a standard technique for evaluating new implants since early migration can predict loosening $[9$, $16,24]$. The RSA method in our study followed published guidelines for RSA [28] and the method developed for this particular implant [27]. Using this method the accuracy is between 0.08 and $0.19 \mathrm{~mm}$ and the precision $0.12-0.33 \mathrm{~mm}$. During surgery 5-6 tantalum markers $(1.0 \mathrm{~mm})$ were placed in the bone surrounding the implant to serve as the reference segment for the RSA analysis. The tip of the implant, in the shape of a 3-mm hemi-sphere was used as the measured point of the prosthesis. Two days postoperatively, at 6 months, 1 and 2 years after surgery, the operated knee was then placed in a biplanar calibration cage (Cage 10; RSA Biomedical AB, Umeå, Sweden). Digital radiographs (Bucky Diagnostic; Philips, Eindhoven, the Netherlands) 
were then taken using one fixed and one mobile X-ray source. The exposure was set to $125 \mathrm{kV}$ and $2.5 \mathrm{mAs}$. The radiographs were saved in a standard dicom file format (resolution $254 \mathrm{dpi}$ ) and uploaded to a workstation. UmRSA 6.0 computer software (RSA Biomedical) was used for all measurements and migration analyses. The markers in the distal femur form one segment and the micromotion of the tip of the implant was then evaluated. The 3D translations of the tip in relation to the femoral bone segment were calculated at each follow-up visit and compared with the immediate postoperative measurements. We also measured the maximum total point movement (MTPM), which is the 3D translation vector of the tip. At 1 year, we performed two examinations 15 min apart on all patients with complete repositioning of the X-ray tubes and the calibration cage. We calculated the precision as the $95 \%$ confidence interval (SD 1.96) of the difference between these examinations. For translation along the $x$-(transverse), $y$-(vertical) and $z$-[anteroposterior (AP)] axes, this was $0.20,0.32$ and $0.30 \mathrm{~mm}$, respectively, and for the MTPM it was $0.33 \mathrm{~mm}$, corresponding well with previous in vitro precision determinations [27]. For individual patients, any migrations above these threshold values means that a detectable migration has occurred of the implant in relation to the bone.

The study was approved by the local ethics committee Karolinska Institutet (2012/109-3171). Informed consent was obtained from all patients and the study was conducted in accordance with good clinical practice and the declaration of Helsinki.

\section{Statistical analysis}

All data were analyzed using IBM SPSS Statistics for Mac, version 23 (IBM Corp, Armonk, NY, USA). Data for patient demographics and patient-related outcome measures are expressed as median and range. Group differences were analyzed with Mann-Whitney rank sum test, two-tailed. A $p$ value of less than 0.05 was considered statistically significant.

\section{Results}

\section{Patients}

Thirteen of the screened patients fulfilled the inclusion criteria. Two patients chose not to proceed with surgery. On ethical grounds questions about reasons for drop-out could not be asked. For one patient the injury was per-operatively found to be more severe than anticipated, $24 \mathrm{~mm}$, and could not be sufficiently replaced by the planned implant, $20 \mathrm{~mm}$, and was therefore excluded. The remaining ten patients were followed for 2 years. Demographics, injury and surgical data as described in Table 1 with median age of 42.5 years (range 36-56), seven male and three female patients, seven ICRS grade 4 and three ICRS grade 3 injuries, implant size $17 \mathrm{~mm}$ in four patients and $20 \mathrm{~mm}$ in six patients. All surgeries were done on the medial femoral condyle. All patients had previous surgery in the involved knee. Seven patients had previous failed microfracture and three patients had previously had ACL reconstruction and minor medial meniscus injuries with small flap tears that were resected. If occupations with low physical demand, sick leave was short, but sick leave was necessary for up to 1 year with high demanding manual labor. Patient no. 6 was unemployed at time of surgery, but found work as a janitor 6 months after surgery.

\section{Surgical usability of implant and instruments}

Surgery time improved with increased experience and each subsequent procedure and reached below 60 min (Table 1). In two patients (nos. 1 and 2) the cartilage injury on the medial femoral condyle was localized lateral and close to the patella and trochlea which made it difficult to position the drillguide. The problem was solved by cutting the most anterolateral part of the drillguide which did not affect the stability of the guide and the continuing procedure.

\section{Radiographic evaluation}

Radiographs at 2 years showed no peri-prosthetic radiolucency, cyst formation, implant subsidence or other signs of disassembly. No radiographic sign of damage to the opposing tibial cartilage or signs of osteoarthritis such as decreased joint space was observed (Fig. 2).

\section{Radiostereometric analysis}

There were no missing RSA data on any of the follow-ups. Overall, we found very small migration and no implant migrated beyond the detection limit within 6 months postoperatively. After this period, one implant migrated between the 6 months and 1 year follow-up in the $z$-axes, but had stabilized at the 2-year follow-up. The mean overall migration was statistically significant compared to the postoperative value for MTPM but not for individual migrations in the $x-y$ or $z$-axes (Table 2).

\section{Patient-related outcome measures and range of motion measurements}

At 2 years all follow-up scores; VAS, EQ5D and KOOS showed improvements that reached significance for VAS ( $p$ $\leq 0.001)$, Tegner $(p=0.034)$, the KOOS subscores ADL $(p=0.0048)$, sport and recreation $(p=0.034)$ and quality of life $(p=0.037)$. VAS and KOOS scores improved 


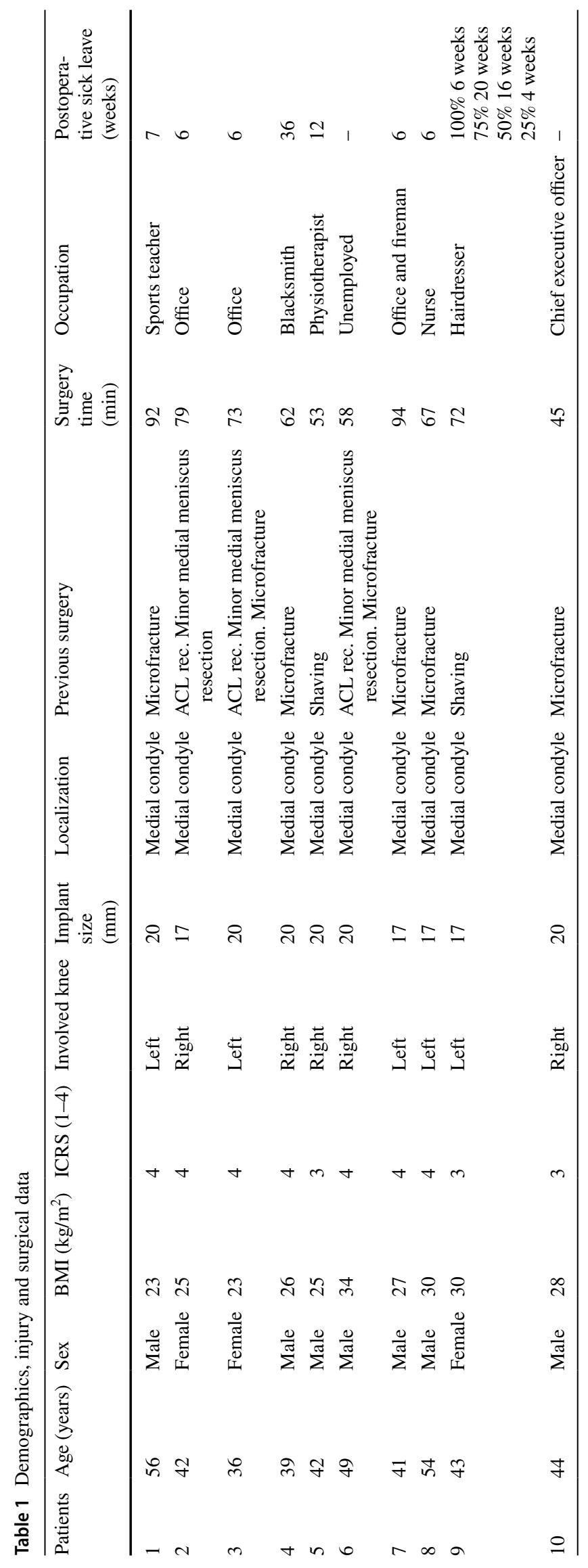





Fig. 2 Radiographs, 2 years, patient no. 4

gradually, with the greatest improvement between month 3 and 6 (Figs. 3, 4). The improvements in EQ5D did not reach statistical significance. No infections, deep venous thrombosis or other complications occured in the postoperative period. A second-look arthroscopy was performed in patient no. 2, 10 months after the index surgery due to persistent anterior knee pain. The arthroscopy showed slight patellofemoral osteoarthritis, ICRS 1-2. The implant was well fixed and cartilage laid over the edges of the implant. Range of motion reached preoperative values for all patients at 6 weeks post-surgery.

\section{Discussion}

Good subjective outcome and no implant migration were shown in this first clinical report of a new customized focal knee resurfacing system, the Episealer ${ }^{\circledR}$. The middle-aged patients, recently noted as the "gap"-patient [19], with a disabling focal cartilage injury in the knee is a challenging
Table 2 Migration of the proximal tip of the implant measured with RSA compared to the 2-day postoperative value

\begin{tabular}{|c|c|c|c|}
\hline \multirow[t]{2}{*}{ Tip migration (mm) } & \multicolumn{2}{|c|}{ Migration } & \multirow[t]{2}{*}{$p$ value } \\
\hline & Mean & SD & \\
\hline \multicolumn{4}{|l|}{ Transverse $(x)$} \\
\hline 6 months & 0.00 & 0.09 & n.s \\
\hline 1 year & -0.09 & 0.27 & n.s \\
\hline 2 years & -0.04 & 0.20 & n.s \\
\hline \multicolumn{4}{|l|}{ Vertical $(y)$} \\
\hline 6 months & -0.03 & 0.12 & n.s \\
\hline 1 year & 0.03 & 0.26 & n.s \\
\hline 2 years & 0.00 & 0.20 & n.s \\
\hline \multicolumn{4}{|l|}{ Anteroposterior $(z)$} \\
\hline 6 months & 0.03 & 0.10 & n.s \\
\hline 1 year & -0.12 & 0.36 & n.s \\
\hline 2 years & -0.06 & 0.15 & n.s \\
\hline \multicolumn{4}{|l|}{ МТРM } \\
\hline 6 months & 0.16 & 0.09 & $<0.001$ \\
\hline 1 year & 0.32 & 0.43 & 0.04 \\
\hline 2 years & 0.27 & 0.16 & $<0.001$ \\
\hline
\end{tabular}

group. Common cartilage procedures have shown inferior results in this group of patients and a conventional arthroplasty poses great risk of need for revision surgery due to implant loosening and wear [11, 13-15, 23]. The patients reported here had had a number of previous procedures during long periods of time and were in a salvage situation. It was shown that the Episealer ${ }^{\circledR}$ device was an effective treatment option for focal cartilage lesions in these highly symptomatic middle-aged patients. The short-term patientrelated outcome measures showed good-to-excellent results and was in accordance with previously reported results from other focal knee resurfacing systems [7, 29]. Further followup to ensure continued good clinical results at long-term is however mandatory.

The purpose of the study was primarily to ensure good implant safety and surgical usability of the implant and instruments. In two patients the drill guide was hard to position since the cartilage defect was in close proximity to the patella and trochlea. Trimming the anterolateral part of the guide was necessary and that allowed good guide stability and positioning. The surgeries were performed by experienced knee surgeons, but it should be noted that there is a learning curve and surgery time decreased with each subsequent procedure. Surgery time was affected by the study protocol, check list procedures and thorough documentation necessary to ensure good patient safety with this new procedure.

In one patient MRI underestimated the size of the injury and the implant was to small to fit the damaged site. A reassessment of the MRI examination was made postoperatively 
Fig. 3 KOOS subscales at preop, 3, 6, 12 and 24 months
Fig. 4 VAS at pre-op, 1, 2 and 6 weeks. $3,6,12$ and 24 months
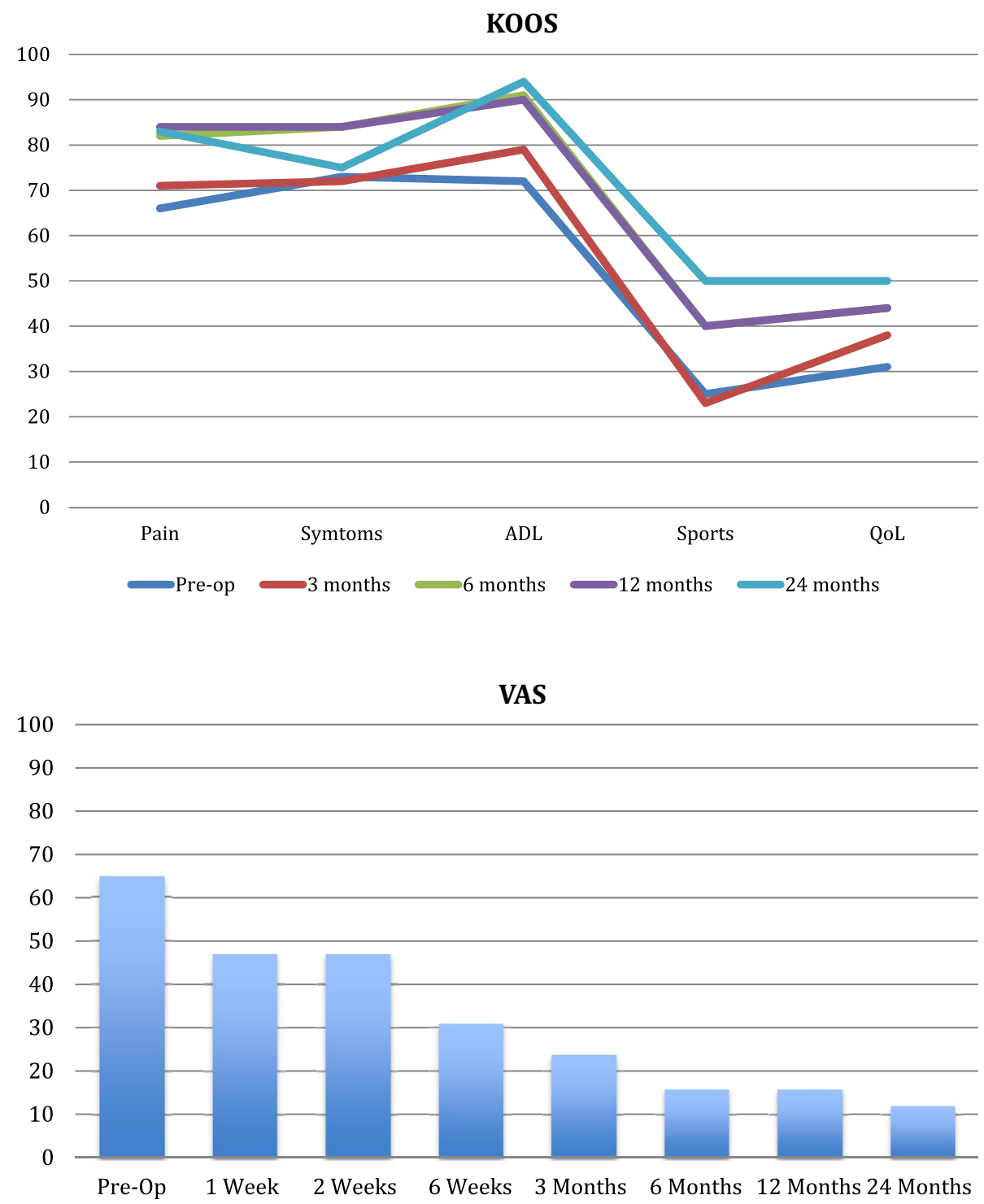

and this prompted changes in the MRI evaluation procedures and no incidents with implants not matching the site of the injury has occured thereafter.

In one patient a second-look arthroscopy was performed after 10 months. The implant showed good osteochondral integration and no signs of implant-related injuries to the adjacent tibia plateau. The prosthesis appeared well incorporated.

To our knowledge, there are no previous publications on RSA and knee resurfacing implants. The migration of our implant is well below (non-detectable) the proposed limit of $0.3 \mathrm{~mm}$, which is predictive of late failure of a total knee prosthesis [24]. Only one patient had migration up until 1 year, but was stable after 2 years. This indicates that the implant is stable enough under an extended period of time for osseo-integration to occur. The limitations of our RSA method when measuring only the tip of the implant as a proxy for overall migration, has been well described in the methods paper for this prosthesis [27]. To acquire more thorough migration measurements, including rotations, newly developed methods using three-dimensional computed tomography RSA would be required for these types of implants [4]. At the initiation of the current study, this was not available but can possibly be used in future studies where small implants like these are used and where RSA marking is not possible.

Radiographic exams at 12 months showed no signs of tibial damage or progressing osteoarthritis. It is recognized that focal knee resurfacing with metal implants warrants correct positioning in order to avoid injuries to the opposing tibia and subsequent cartilage degeneration [2, 20]. Previous mid-term follow-up of focal knee resurfacing with metal implants have shown risk of osteoarthritis progression with more than $20 \%$ revision to knee arthroplasty within 7 years 
[17]. A similar focal knee resurfacing method with a metal implant has shown progressing radiographic Kellgren-Lawrence osteoarthritis scores even at short-term follow-up [8]. However, it has been stipulated that correct implant placement does not lead to increased contact pressures $[1,3]$. The purpose with the development of an individualized implant and instrumentation system was to achieve accurate and consistent implant position at a correct angle and depth, thereby minimizing the risk for a misaligned and protruding implants in order to avoid further cartilage damage with the expectation to maintain the good results even at long-term.

\section{Conclusion}

This new customized focal knee resurfacing implant shows good implant safety and patient satisfaction, function as well as pain is significantly improved. RSA did not detect any implant migration. A limitation of this study is that it is a short-term follow-up and it must be emphasized that the small group of patients do not allow generalization of the results and further follow-up and research is mandatory. The implant should be used cautiously by experienced surgeons.

\section{Compliance with ethical standards}

Funding Episurf $\mathrm{AB}$ has provided the prosthesis and instruments. Research fundings were from Sophiahemmet, Stockholm and Capio Research funds.

Conflict of interest AS, MH, DR and NM has no conflicts of interest. $\mathrm{LR}$ is a senior consultant and board member for Episurf AB.

Ethical approval The study was approved by the local ethics committee Karolinska Institutet (2012/109-3171).

Informed consent Informed consent was obtained from all patients and the study was conducted in accordance with good clinical practice and the declaration of Helsinki.

Open Access This article is distributed under the terms of the Creative Commons Attribution 4.0 International License (http://creativecommons.org/licenses/by/4.0/), which permits unrestricted use, distribution, and reproduction in any medium, provided you give appropriate credit to the original author(s) and the source, provide a link to the Creative Commons license, and indicate if changes were made.

\section{References}

1. Becher C, Huber R, Thermann H, Ezechieli L, Ostermeier S, Wellmann M, von Skrbensky G (2011) Effects of a surface matching articular resurfacing device on tibiofemoral contact pressure: results from continuous dynamic flexion-extension cycles. Arch Orthop Trauma Surg 131(3):413-419

2. Becher C, Huber R, Thermann H, Paessler HH, Skrbensky $\mathrm{G}$ (2008) Effects of a contoured articular prosthetic device on tibiofemoral peak contact pressure: a biomechanical study. Knee Surg Sports Traumatol Arthrosc 16(1):56-63

3. Bollars P, Bosquet M, Vandekerckhove B, Hardeman F, Bellemans J (2012) Prosthetic inlay resurfacing for the treatment of focal, full thickness cartilage defects of the femoral condyle: a bridge between biologics and conventional arthroplasty. Knee Surg Sports Traumatol Arthrosc 20(9):1753-1759

4. Broden C, Olivecrona H, Maguire GQ Jr, Noz ME, Zeleznik MP, Skoldenberg O (2016) Accuracy and precision of three-dimensional low dose CT compared to standard RSA in acetabular cups: an experimental study. BioMed Res Int 2016:5909741

5. Chahal J, Thiel GV, Hussey K, Cole BJ (2013) Managing the patient with failed cartilage restoration. Sports Med Arthrosc 21(2):62-68

6. Cicuttini F, Ding C, Wluka A, Davis S, Ebeling PR, Jones G (2005) Association of cartilage defects with loss of knee cartilage in healthy, middle-age adults: a prospective study. Arthritis Rheum 52(7):2033-2039

7. Curl WW, Krome J, Gordon ES, Rushing J, Smith BP, Poehling GG (1997) Cartilage injuries: a review of 31,516 knee arthroscopies. Arthroscopy 13(4):456-460

8. Dhollander AA, Almqvist KF, Moens K, Vandekerckhove PJ, Verdonk R, Verdonk P, Victor J (2015) The use of prosthetic inlay resurfacing as a salvage procedure for a failed cartilage repair. Knee Surg Sports Traumatol Arthrosc 23(8):2208-2212

9. Hansson U, Toksvig-Larsen S, Ryd L, Aspenberg P (2009) Onceweekly oral medication with alendronate does not prevent migration of knee prostheses: a double-blind randomized RSA study. Acta Orthop 80(1):41-45

10. Heir S, Nerhus TK, Røtterud JH, Løken S, Ekeland A, Engebretsen L, Arøen A (2010) Focal cartilage defects in the knee impair quality of life as much as severe osteoarthritis: a comparison of knee injury and osteoarthritis outcome score in 4 patient categories scheduled for knee surgery. Am J Sports Med 38(2):231-237

11. Hunziker EB, Lippuner K, Keel MJ, Shintani N (2015) An educational review of cartilage repair: precepts \& practice e myths $\&$ misconceptions e progress \& prospects. Osteoarthr Cartil 23(3):334-350

12. Jungmann PM, Salzmann GM, Schmal H, Pestka JM, Südkamp NP, Niemeyer P (2012) Autologous chondrocyte implantation for treatment of cartilage defects of the knee-what predicts the need for reintervention. Am J Sports Med 40(1):58-67

13. Knutsen G, Drogset JO, Engebretsen L, Grøntvedt T, Isaksen V, Ludvigsen TC, Roberts S, Solheim E, Strand T, Johansen O (2007) A randomized trial comparing autologous chondrocyte implantation with microfracture, findings at five years. J Bone Joint Surg Am 89(10):2105-2112

14. Knutsen G, Engebretsen L, Ludvigsen TC, Drogset JO, Grøntvedt T, Solheim E, Strand T, Roberts S, Isaksen V, Johansen O. Drogset JO, Grøntvedt T, Solheim E, Strand T, Roberts S, Isaksen V, Johansen O (2004) Autologous chondrocyte implantation compared with microfracture in the knee. A randomized trial. J Bone Joint Surg Am 86:455-464

15. Kreuz PC, Erggelet C, Steinwachs MR, Krause SJ, Lahm A, Niemeyer P, Ghanem N, Uhl M, Südkamp N (2006) Is microfracture of chondral defects in the knee associated with different results in patients aged 40 years or younger? Arthroscopy 22(11):1180-1186

16. Kärrholm J, Borssen B, Lowenhielm G, Snorrason F (1994) Does early micromotion of femoral stem prostheses matter? 4-7-year stereoradiographic follow-up of 84 cemented prostheses. J Bone Joint Surg Br 76(6):912-917

17. Laursen JO, Lind M (2017) Treatment of full-thickness femoral cartilage lesions using condyle resurfacing prosthesis. Knee Surg Sports Traumatol Arthrosc 25(3):746-751 
18. Lefkoe TP, Trafton PG, Ehrlich MG, Walsh WR, Dennehy DT, Barrach HJ (1993) An experimental model of femoral condylar defect leading to osteoarthrosis. J Orthop Trauma 7(5):458-467

19. Li CS, Karlsson J, Winemaker M, Sancheti P, Bhandari M (2014) Orthopedic surgeons feel that there is a treatment gap in management of early OA:international survey. Knee Surg Sports Traumatol Arthrosc 22(2):363-378

20. Martinez-Carranza N, Berg HE, Hultenby K, Nurmi-Sandh H, Ryd L, Lagerstedt AS (2013) Focal knee resurfacing and effects of surgical precision on opposing cartilage. Osteoarthr Cartil 21(5):739-745

21. Martinez-Carranza N, Berg HE, Lagerstedt AS, Nurmi-Sandh H, Schupbach P, Ryd L (2014) Fixation of a double-coated titaniumhydroxyapatite focal knee resurfacing implant:a 12-month study in sheep. Osteoarthr Cartil 22(6):836-844

22. Martinez-Carranza N, Ryd L, Hultenby K, Hedlund H, NurmiSandh H, Lagerstedt AS, Schupbach P, Berg HE (2016) Treatment of full thickness focal cartilage lesions with a metallic resurfacing implant in a sheep animal model, 1 year evaluation. Osteoarthr Cartil 24(3):484-493

23. Meehan JP, Danielsen B, Kim SH, Jamali AA, White RH (2014) Younger age is associated with a higher risk of early periprosthetic joint infection and aseptic mechanical failure after total knee arthroplasty. J Bone Joint Surg Am 96(7):529-535

24. Ryd L, Albrektsson BE, Carlsson L, Dansgard F, Herberts P, Lindstrand A, Regner L, Toksvig-Larsen S (1995) Roentgen stereophotogrammetric analysis as a predictor of mechanical loosening of knee prostheses. J Bone Joint Surg Br 77(3):377-383

25. Shapiro F, Koide S, Glimcher MJ (1993) Cell origin and differentiation in the repair of full-thickness defects of articular cartilage. J Bone Joint Surg Am 75:532-553

26. Schinhan M, Gruber M, Vavken P, Dorotka R, Samouh L, Chiari C, Gruebl-Barabas R, Nehrer S (2012) Critical-size defect induces unicompartmental osteoarthritis in a stable ovine knee. J Orthop res 30(2):214-220

27. Sköldenberg O, Eisler T, Stark A, Muren O, Martinez-Carranza N, Ryd L (2014) Measurement of the migration of a focal knee resurfacing implant with radiostereometry. Acta Orthop 85(1):79-83

28. Valstar ER, Gill R, Ryd L, Flivik G, Börlin N, Kärrholm J (2005) Guidelines for standardization of radiostereometry (RSA) of implants. Acta Orthop 76(4):563-572

29. Widuchowski W, Widuchowski J, Trzaska T (2007) Articular cartilage defects: study of 25,124 knee arthroscopies. Knee 14(3):177-182 